\title{
EL POLEN RECOGIDO POR APIS MELLIFERA L. EN HINOJOS (HUELVA) DURANTE LA PRIMAVERA
}

\author{
Pedro L. ORTIZ
}

\begin{abstract}
RESUMEN. El polen recogido por Apis mellifera L. en Hinojos (Huelva) durante la primavera. Durante la primavera de 1986, periódicamente, se ha usado una trampilla cazapolen para obtener muestras de las cargas de polen recogidas por dos colmenas instaladas en Hinojos (Huelva). Dichas muestras se han pesado y se han estudiado microscópicamente. En cada una se han identificado los tipos polínicos encontrados y se han calculado sus porcentajes respecto al volumen; además se ha calculado un índice proporcional a la cantidad de cada tipo polínico recogida cada día de muestreo. Se han encontrado 53 tipos polínicos diferentes, pero más de la mitad del volumen de cada muestra correspondió a uno o dos tipos solamente. En general los tipos recogidos abundantemente por una colmena también lo fueron por la otra. Los recursos polínicos más usados fueron Cistus salvifolius, C. libanotis y Quercus suber. El índice de cantidad y el porcentaje respecto a volumen de cada tipo polínico no evolucionaron paralelamente a lo largo del muestreo.
\end{abstract}

Palabras clave. Polen, palinología, Huelva, España, Apis mellifera, cargas de polen.

ABSTRACT. The pollen collected by Apis mellifera L. in Hinojos (Huelva) during the spring. During the spring of 1986, periodically, pollen loads were trapped from two honeybee colonies located in Hinojos (Huelva). Pollen load samples were weighed and studied microscopically. Pollen types found in each sample were identified and their percentages of volume calculated; moreover, an index proportional to the amount of each pollen type collected each sampling day was calculated. 53 pollen types were found, but more than $50 \%$ of the volume of each sample belonged to one or two types only. Most pollen types collected in abundance by one of the colonies were also collected by the other one. The main pollen sources were Cistus salvifolius, C. libanotis and Quercus suber. The index of amount and the percentage of volume of each pollen type did not evolve parallel throughout the sampling period.

Key words. Pollen, palynology, Huelva, Spain, Apis mellifera, pollen loads.

\section{INTRODUCCIÓN}

El análisis microscópico de muestras de cargas de polen recogidas por las abejas es un instrumento eficaz para estudiar la dieta polínica de las mismas y las relaciones de éstas con la flora de un territorio (Synge, 1947;
Percival, 1947, 1950, 1955; p.e.). Este análisis ha sido abordado por distintos autores siguiendo básicamente dos métodos diferentes, con diversas variantes. Uno de ellos consiste en separar las cargas de polen de cada muestra en grupos homogéneos de acuerdo con sus caracteres externos e identificar microscópicamente 
el polen presente en cada uno de ellos (Louveaux, 1958a; McLellan, 1976; Zuccoli, 1987; Parent et al., 1990; p.e.). Esta manera de proceder es muy laboriosa, y la separación de cargas de diferente origen botánico plantea dificultades, a veces insalvables (Hidalgo Berutich y Bootello Bravo, 1990). La otra metodología, bastante menos trabajosa, consiste en obtener una suspensión homogénea de la muestra, y observar parte de ella al microscopio para identificar los tipos polínicos presentes y calcular la frecuencia de aparición de cada uno de ellos (Ramalho y KleinertGiovannini, 1896; Bolchi Serini, 1986-87; Pellin et al., 1990; p.e.). Además, varios autores han señalado que cuando se emplea este segundo método es conveniente introducir factores de corrección que tengan en cuenta las diferencias de volumen de los granos de los distintos tipos polínicos (Buchmann y O'Rourke, 1991; Biesmeijer et al., 1992); de este modo se conoce qué proporción del volumen de la muestra corresponde a cada tipo polínico. Evidentemente, y tal como señalan los citados autores, las proporciones respecto a volumen constituyen una medida más real del polen recogido por las abejas que las frecuencias respecto al número total de granos de cada muestra.

Sin embargo, este tipo de trabajos no suelen considerar la cantidad real de polen de la que es representativa cada muestra estudiada. Dicha cantidad puede ser tremendamente variable dada la estacionalidad de la actividad recolectora de polen de Apis mellifera, al menos en lugares de clima templado (Louveaux, 1958a; McLellan, 1978; Ortiz, 1991); por ello, en este estudio, se ha creído oportuno tener en cuenta este factor.

El presente trabajo pretende conocer las fuentes de polen utilizadas por las abejas en Hinojos (Huelva) durante la primavera y comparar los resultados expresados en porcentajes de volumen de cada muestra con los obtenidos si se consideran las cantidades de polen de las que son representativas dichas muestras.

\section{MATERIAL Y MÉTODOS}

Se han utilizado dos colmenas tipo Langstroth instaladas en Hinojos (Huelva) en un área de pinar (Pinus pinea $\mathrm{L}$.) con restos de alcornocal (Quercus suber L.) y abundante matorral rico en Cistaceae, Fabaceae y Lamiaceae; existen también en las inmediaciones de las colmenas algunos cultivos de Eucalyptus spp. y espacios desprovistos de arbolado y matorral donde prosperan comunidades herbáceas importantes.

Se han obtenido muestras del polen recogido por ambas colonias durante la primavera de 1986. Para ello se colocó una trampilla cazapolen cada semana en la entrada de una de las colmenas alternativamente. La trampilla se colocaba al anochecer, cuando todas las pecoreadoras habían regresado, y se retiraba 24 ó 48 horas después dependiendo de la actividad observada. Las muestras de la trampilla se han deshidratado en una cámara secadora por corriente de aire caliente a $40-45^{\circ} \mathrm{C}$ (Jean-Prost, 1985) y se han pesado.

Posteriormente, las muestras de polen obtenidas en las trampillas han sido analizadas microscópicamente. De cada una de ellas se ha tomado una submuestra de 5 gramos y se han dispersado sus cargas formando una pasta homogénea con ácido acético, a partir de la cual se han elaborado cuatro preparaciones microscópicas empleando el método acetolítico de Erdtman (1960) con algunas modificaciones.

Se han contado e identificado al menos 300 granos de polen en cada muestra (al menos 75 en cada preparación) para determinar la frecuencia de cada tipo polínico respecto al número total de granos de polen en la muestra. A partir de estas frecuencias y del volumen medio de un grano de cada tipo, se ha estimado la proporción de cada tipo polínico respecto al volumen total de granos de polen en la muestra. Para calcular el volumen medio de un grano de cada tipo polínico se han usado las medidas de Valdés et al. (1987). 
En cada muestra de polen, el porcentaje respecto a volumen de cada tipo polínico se ha multiplicado por el peso de polen retenido en la trampilla el día de su recogida. A la cifra más elevada obtenida en uno de estos productos, considerando los de todas las muestras de ambas colmenas, se le ha asignado el valor 100 y a las restantes se les han dado valores proporcionales. Así se ha obtenido el que hemos llamado «índice de cantidad», cuyo valor, para cada tipo polínico presente en una muestra, es mayor que 0 y menor o igual que 100 , y es indicativo del volumen de polen de ese tipo acumulado en la trampilla el día que se retiró esa muestra. Se ha comprobado previamente que el peso de cada muestra de polen es directamente proporcional al volumen que la misma ocupa en una probeta.

\section{RESULTADOS}

La cantidad de polen recogida por la colmena 1 en un día de muestreo osciló entre 10

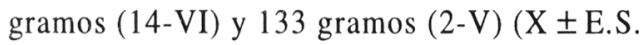
$=51.4 \pm 15.88 ; n=7)$. En la colmena 2 varió desde 4.5 gramos (7-VI) hasta 232 gramos $(11-I V)(X \pm$ E.S. $=89.57 \pm 33.49 ; \mathrm{n}=7)$.

En el conjunto de muestras estudiadas se han encontrado 53 tipos polínicos diferentes, 44 en las de la colmena 1 y 45 en las procedentes de la 2, siendo 36 de ellos comunes a ambas. El número de tipos por muestra oscila entre 6 y $20(\mathrm{X} \pm$ E.S. $=12.86 \pm 1.61 ; \mathrm{n}=7)$ en la colmena 1 y entre 6 y $18(X \pm$ E.S. $=13.29 \pm$ $1.49 ; \mathrm{n}=7$ ) en la 2 . Sin embargo, más de la mitad del volumen de cualquiera de las muestras de ambas colmenas corresponde a uno o dos tipos solamente. Las Tablas 1 y 2 muestran los resultados más significativos obtenidos en los análisis de las muestras de las colmenas 1 y 2 respectivamente. En ellas se han incluído todos los tipos polínicos que presentan un porcentaje respecto a volumen superior a 150 un índice de cantidad mayor o igual que 2 en al menos una muestra. Pistacia lentiscus es el único de estos tipos polínicos que ha sido recogido por una sola colmena.

En la colmena 1, la mayor cantidad de polen de un tipo recogida en un día de muestreo correspondió a Quercus suber (2-V). Le siguió en importancia la cantidad recogida de Cistus salvifolius el día 4-IV; además el polen de esta especie fue recogido en cantidades importantes los dos días de muestreo siguientes, por lo que en el conjunto de la primavera fue el tipo polínico más recogido por esta colmena. En esta colonia, son destacables asimismo las cantidades de polen recolectadas en un día de Salix alba (4-IV), Cistus ladanifer (2-V) y Cistus libanotis (17-V). Por otra parte, el polen de Cistus salvifolius constituye más de la mitad del volumen de las muestras de 4 y 19 de Abril y lo mismo ocurre con el de Cistus libanotis en la de 17 de Mayo. Asimismo son destacables los porcentajes respecto a volumen de Raphanus raphanistrum y Oxalis pescaprae en la muestra de Marzo, el de Salix alba (4-IV),el de Cistus ladanifer (19-IV), los de Quercus suber y Cistus salvifolius (2-V), los de Cistus sp. (C. albidus, C. crispus) y Echium plantagineum (31-V) y los de Eucalyptus spp., Halimium halimifolium y Myrtus communis (14-VI). No obstante, en las muestras de 21-III, 31-V y 14-VI, ninguno de los tipos polínicos destacados por su porcentaje respecto a volumen alcanza un índice de cantidad elevado.

La mayor cantidad de polen recogida en un día de un solo tipo por la colmena 2 correspondió a Cistus salvifolius (11-IV), siendo igualmente muy importante la cantidad recogida de esta especie el siguiente día de muestreo, por lo que en el conjunto de la estación fue el principal recurso polínico, también para esta colonia. Además, en la colmena 2 son reseñables por su cantidad la recolección de polen de Halimium commutatum, el 11 de Abril, y las de Cistus libanotis y Quercus suber, el 10 de Mayo. Por otro lado, más de la 


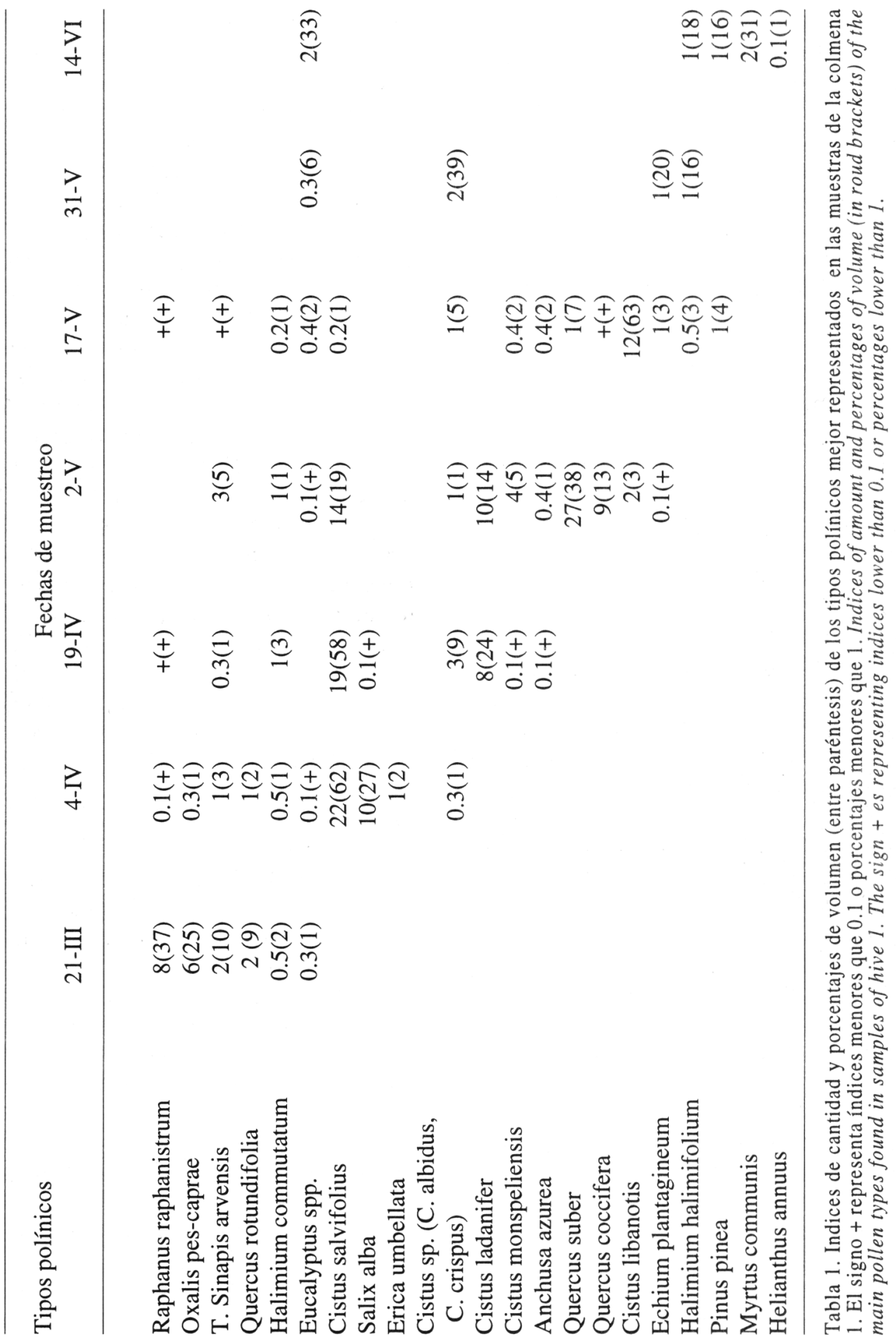




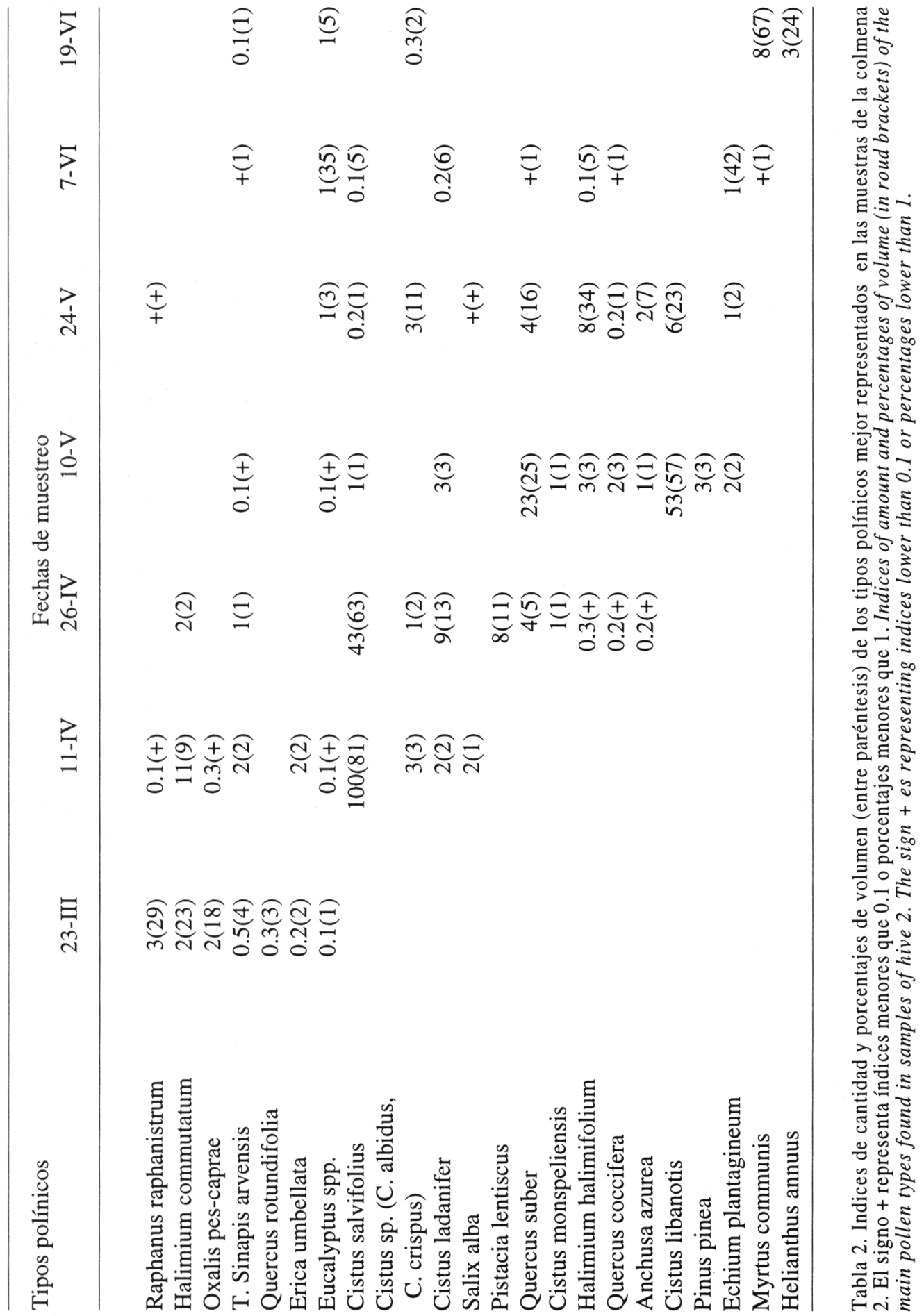


mitad del volumen de las muestras de 11 y 26 de Abril procede de Cistus salvifolius; lo mismo sucede con Cistus libanotis y Myrtus communis en las muestras de $10-\mathrm{V}$ y $19-\mathrm{VI}$, respectivamente. En la muestra de Marzo, el polen de Raphanus raphanistrum, el de Halimium commutatum y el de Oxalis pescaprae están presentes con porcentajes respecto a volumen importantes; lo mismo ocurre con el polen de Quercus suber (10-V), Halimium halimifolium y Cistus libanotis (24V), Eucalyptus spp. y Echium plantagineum (7-VI) y Helianthus annuus (19-VI). Sin embargo, los tipos polínicos destacables por su porcentaje respecto a volumen en las muestras de 23-III, 24-V, 7-VI y 19-VI, no lo son por su índice de cantidad.

En ambas colmenas se observa que el índice de cantidad y el porcentaje respecto a volumen de cada tipo polínico no evolucionan paralelamente a lo largo del muestreo. Una variación del porcentaje de volumen de un tipo en una muestra con respecto al muestreo anterior supone un cambio en el índice de cantidad en el mismo sentido en unos casos y en sentido opuesto en otros. Además, cuando ambos parámetros cambian en el mismo sentido, el orden de magnitud de esa variación es diferente para cada uno de los dos en la mayoría de los casos.

\section{DISCUSIÓN}

Nuestros resultados muestran que en un día determinado el número de especies de las que una colmena obtiene polen es relativamente bajo, siendo muy reducido el de aquéllas de las que lo obtiene en proporciones considerables. Durante el periodo de muestreo se ha estudiado la fenología de la floración en el área de trabajo (Ortiz, 1991). Los resultados de dicho estudio revelan que en todo momento el número de especies en flor en el área de pecoreo de las colmenas es muy superior al de especies utilizadas por las mismas como fuentes de polen. Estas observaciones están en consonancia con el hecho bien conocido de que cada día las abejas de una colonia trabajan sólo en un reducido número de fuentes de alimento localizadas en parcelas concretas (Visscher y Seeley, 1982; p.e.).

Por otra parte, nuestros resultados muestran que para la mayoría de las especies cuyo polen es recogido con cierta importancia por una colmena en uno de los muestreos, esa recogida se mantiene durante varios muestreos consecutivos, mediando alrededor de dos semanas entre cada uno y el siguiente. Esto significa que cuando una colonia selecciona una especie como fuente de polen suele mantenerla como tal durante un periodo considerable, cuya extensión está limitada en última instancia por la duración de la floración de esa especie. Sin duda, este comportamiento de la colmena es un reflejo de la tendencia de cada abeja a permanecer fiel a la fuente de alimento que está utilizando (Butler, 1945; Ribbands, 1949; Singh,1950).

Como consecuencia del bajo número de especies visitadas en un día por las abejas de una colonia y de la fidelidad de éstas a las primeras, y como ponen de manifiesto nuestros resultados, las abejas visitan, a lo largo de la estación, sólo una parte de la flora a su alcance, siendo el número de especies utilizadas intensamente muy reducido si se compara con el de las que florecen en el área de pecoreo de las abejas. Diversos autores han obtenido con anterioridad resultados similares (Percival, 1947; Synge, 1947; Louveaux, 1958b; McLellan, 1976). Además, cabe señalar que las preferencias polínicas de nuestras dos colmenas son bastante parecidas, ya que entre las fuentes de polen elegidas por cada una de ellas existe un alto grado de coincidencia.

Dado que la cantidad de polen recogido diariamente es muy variable a lo largo de la estación, porcentajes respecto a volumen similares en diferentes muestras corresponden a 
cantidades reales de polen desiguales, o incluso muy dispares. Por lo tanto, tal como se ha puesto de manifiesto en nuestros resultados, la valoración de las diferentes especies como componentes de la dieta polínica de las abejas en un periodo determinado varía sensiblemente según se consideren sus porcentajes respecto al volumen de cada muestra o sus cantidades reales recogidas cada día (peso o volumen, o algún índice proporcional adecuado). Si dicha valoración se hace de acuerdo con los porcentajes del volumen, se infravaloran las especies utilizadas en los momentos de mayor intensidad de recolección y se sobrevaloran las usadas cuando el nivel de recogida es menor. Lógicamente la cantidad, calidad y variedad de las reservas de polen, y en último término la dieta polínica de una colonia depende más de las cantidades reales recogidas de cada especie que de los porcentajes que éstas representan en la recolección de cada día. Igualmente, nuestros resultados muestran que si mediante análisis microscópico de muestras de polen se pretende conocer el patrón de utilización de un recurso polínico o la intensidad de visitas de Apis mellifera a una especie determinada durante cierto periodo, los porcentajes de su polen respecto al volumen de las muestras pueden inducir a interpretaciones erróneas. Por tanto, es preferible usar las cantidades reales de cada tipo polínico o algún índice de las mismas, en lugar de los porcentajes del volumen, para estudiar la dieta polínica de colonias de abejas, el patrón de uso de alguna fuente de polen o la intensidad de las visitas a alguna especie mediante análisis polínico de muestras de polen. Para ello es necesario saber de qué cantidad de polen es representativa cada una de las muestras analizadas.

De acuerdo con nuestros resultados y las consideraciones anteriores, Cistus salvifolius es la principal fuente de polen primaveral en el área de estudio; asimismo Cistus libanotis, Quercus suber y Cistus ladanifer son recursos polínicos destacables, y en menor medida tam- bién lo son Halimium commutatum, Halimium halimifolium y Salix alba.

AGRADECIMIENTOS. El autor desea expresar su agradecimiento al Prof. Dr. S. Talavera y la Dra. M. J. Díez por sus comentarios, sugerencias y ayuda. El presente trabajo ha sido financiado con cargo al proyecto de la CICYT PA 85-297.

\section{BIBLIOGRAFÍA}

BIESMEIJER, J.C., B. VAN MARWIJK, K. VAN DEURSEN, W. PUNT \& M.J. SOMMEIJER 1992- Pollen sources for Apis mellifera L (Hym, Apidae) in Surinam, based on pollen grain volume estimates. Apidologie, 23: 245-256.

BOLCHI SERINI, G. -1986/87- Osservazioni qualitative sui bottini di polline di Apis mellifera L. in frutteti. Boll. Zool. agr. Bachic. Ser. II, 19: 99-110.

BUCHMANN, S.L. \& M.K. O'ROURKE -1991Importance of pollen grain volumes for calculating bee diets. Grana, 30: 591-595.

BUTLER, C.G. -1945- The influence of various physical and biological factors of the environment on honeybee activity. An examination of the relationship between activity and nectar concentration and abundance. J. Exp. Biol., 21: 5-12.

ERDTMAN, G. -1960- The acetolysis method. A revised description. Svensk. Bot. Tidskr., 54(4): 561-564.

HIDALGO BERUTICH, M.I. y M.L. BOOTELLO BRAVO -1990- About some physical characteristics of pollen loads collected by Apis mellifera L. Apicoltura, 6: 179-191.

JEAN-PROST, P. -1985- Apicultura. Conocimiento de la abeja. Manejo de la colmena. Mundi Prensa. Madrid.

LOUVEAUX, J. -1958a- Recherches sur la récolte du pollen par les abeilles (Apis mellifera L.). Ann. Abeille, 1(III): 113-188.

LOUVEAUX, J. -1958b- Recherches sur la récolte du pollen par les abeilles (Apis mellifera L.) (suite). Ann. Abeille, 1(IV): 197-221.

McLELLAN, A.R. -1976- Factors affecting pollen harvesting by the honeybee. J. Appl. Ecol., 13(3): 801-811. 
McLELLAN, A.R. -1978- Growth and decline of honeybee colonies and inter-relationships of adult bees, brood, honey and pollen. J. Appl. Ecol., 15: 155-161.

ORTIZ, P.L. -1991- Melitopalinología en Andalucia Occidental. Tesis doctoral. Editorial Universidad de Sevilla. Sevilla.

PARENT, J., M.J. FELLER-DEMALSY \& P.J.H. RICHARD -1990- Les sources de pollen et de nectar dans la région de Rimouski, Québec, Canada. Apidologie, 21: 431-445.

PELLIN, P.P., J.M. FLORES, M. PERALTA, F. PUERTA, M. BUSTOS y F. PADILLA -1990Aportes polínicos invernales en colmenas de Sierra Morena (Córdoba, España). An. Asoc. Palinol. Leng. Esp., 5: 63-69.

PERCIVAL, M. -1947- Pollen collection by Apis mellifera. New Phytol., 46: 142-173.

PERCIVAL, M. -1950- Pollen presentation and pollen collection. New Phytol., 49: 40-63.

PERCIVAL, M. -1955- The presentation of pollen in certain Angiosperms and its collection by Apis mellifera. New Phytol., 54: 353-368.

RAMALHO, M. \& A. KLEINERT-GIOVANNINI 1986- Some aspects of the utilization of pollen analysis in ecological research. Apidologie, 17(2): 159-174.

RIBBANDS, C.R. -1949- The foraging method of individual honey-bees. J. Anim. Ecol., 18: 47-66.
SINGH, S. -1950- Behavior studies of honeybees in gathering nectar and pollen. Cornell Univ. Agric. Exp. Sta. Mem., 288: 1-57.

SYNGE, A.D. -1947- Pollen collection by honeybees (Apis mellifera). J. Anim. Ecol., 16: 122-138.

VALDÉS, B., M.J. DÍEZ e I. FERNÁNDEZ -1987Atlas Polínico de Andalucía Occidental. Inst. Des. Regional. y Excma. Diputaciòn de Cádiz. Sevilla.

VISSCHER, P.K. \& T.D. SEELEY -1982- Foraging strategy of honeybee colonies in a temperate deciduous forest. Ecology, 63(6): 1790-1801.

ZUCCOLI, L. -1987- Pollini di piante anemogame ed entomogame bottinati da Apis mellifera L. in bassa Valtellina. Apicoltura, 3: 101-111.

Aceptado para su publicación en Febrero de 1994

Dirección del autor. Departamento de Biología Vegetal y Ecología.Universidad de Sevilla. Apdo. 1095. 41080 Sevilla. 